\title{
Quantifying Particle Numbers and Mass Flux in Drifting Snow
}

\author{
Philip Crivelli $^{1}$ - Enrico Paterna ${ }^{1} \cdot$ Stefan Horender $^{1}$. \\ Michael Lehning ${ }^{1,2}$
}

Received: 26 August 2015 / Accepted: 24 May 2016 / Published online: 22 June 2016

C Springer Science+Business Media Dordrecht 2016

\begin{abstract}
We compare two of the most common methods of quantifying mass flux, particle numbers and particle-size distribution for drifting snow events, the snow-particle counter (SPC), a laser-diode-based particle detector, and particle tracking velocimetry based on digital shadowgraphic imaging. The two methods were correlated for mass flux and particle number flux. For the SPC measurements, the device was calibrated by the manufacturer beforehand. The shadowgrapic imaging method measures particle size and velocity directly from consecutive images, and before each new test the image pixel length is newly calibrated. A calibration study with artificially scattered sand particles and glass beads provides suitable settings for the shadowgraphical imaging as well as obtaining a first correlation of the two methods in a controlled environment. In addition, using snow collected in trays during snowfall, several experiments were performed to observe drifting snow events in a cold wind tunnel. The results demonstrate a high correlation between the mass flux obtained for the calibration studies $(r \geqslant 0.93)$ and good correlation for the drifting snow experiments $(r \geqslant 0.81)$. The impact of measurement settings is discussed in order to reliably quantify particle numbers and mass flux in drifting snow. The study was designed and performed to optimize the settings of the digital shadowgraphic imaging system for both the acquisition and the processing of particles in a drifting snow event. Our results suggest that these optimal settings can be transferred to different imaging set-ups to investigate sediment transport processes.
\end{abstract}

Keywords Cold wind tunnel · Drifting snow · Method comparison - Shadowgraphy · Snow-particle counter

Philip Crivelli

philip.crivelli@slf.ch

1 WSL-Institute for Snow and Avalanche Research SLF, Flüelastrasse 11, 260 Davos Dorf, Switzerland

2 School of Architecture, Civil and Environmental Engineering, Ecole Polytechnique Federal de Lausanne, CRYOS, Lausanne, Switzerland 


\section{Introduction}

Drifting and blowing snow are phenomena that occur often and globally; they have an effect on traffic, when drift accumulations block roads or parts of airports, or when blowing snow leads to poor visibility. Snow transport increases the risk of avalanches in mountainous terrain (Schirmer et al. 2009) or has an impact on mass balances in polar ice sheets and glaciers (Groot Zwaaftink et al. 2013). The physical process behind the drifting of snow is called saltation (see the pioneering work of Bagnold 1935). Much research has been published since then, most often on the saltation of sand. Several methods to quantify particle number flux and mass flux within the atmospheric boundary layer have been described, such as particle counters (Nishimura et al. 1998; Sugiura et al. 1998), flow compartment traps (Schmidt 1986) and methods based on shadowgraphic particle tracking velocimetry (PTV; Gromke et al. 2014). Another approach uses numerical simulations (Doorschot et al. 2004). Generally, similar methods to quantify drifting snow can be applied to many materials, such as sand or other wind-entrained particles. The physical processes are similar for these materials, with differences generally caused by the material properties. The aim of the present study is to quantify drifting snow with two of the most common and most recently used methods, the snow-particle counter (SPC) and shadowgraphic PTV. To the authors' knowledge, no comparison between the SPC method and any other imaging technique has been published. Results of a preliminary study conducted by the authors revealed a very weak correlation between the SPC and the PTV methods. A major problem was that the parameters for the post-processing of the PTV images need appropriate tuning to obtain a reliable estimate of absolute mass-flux values. We present a set of parameters that are appropriate for the PTV method for drifting snow and further explain how results of a pointwise measurement such as the SPC correlate to what can be seen in the images recorded with shadowgraphic PTV. The SPC, which provides total mass flux of snow and the particle-size distribution at a frequency of $1 \mathrm{~Hz}$ is taken as a reference for the mass flux. Based on this (arbitrary) reference, the PTV settings are optimized in order to achieve maximum correlation with the SPC signal. The term arbitrary is used because the SPC can only be calibrated using metal wires of a certain diameter, and not with snow or other similar particles.

\section{Instrumentation and Methods}

The experiments were carried out in the wind tunnel of the WSL Institute for Snow and Avalanche Research, SLF in Davos. The wind tunnel is situated in an unheated concrete building in the Flüelatal, Davos, Switzerland at $1670 \mathrm{~m}$ a.s.l. This particular wind tunnel has been described in Clifton et al. (2006, 2008), Guala et al. (2008), Burri et al. (2011), Gromke et al. (2011), Walter et al. (2012a, b, c, 2014), Pokorny and Horender (2014), Gromke et al. (2014), Paterna et al. (2016). The wind tunnel is $14 \mathrm{~m}$ long, with a $1 \mathrm{~m} \times 1 \mathrm{~m}$ cross-section. At the inlet a honeycomb grid and a 4:1 contraction are placed upstream of the test section while spires mounted on the floor downstream of the contraction create large-scale turbulence. Downstream of the spires, a 6-m region of regular roughness elements is mounted on the floor. The wind tunnel operates in suction mode drawing air through the inlet (refer to Fig. 1a).

Natural snow is collected outside of the wind tunnel in trays during snowfall events. The trays are positioned on the wind-shaded and sun-shaded sides of the wind tunnel such that disturbances during the accumulation of natural snow are reduced. As soon as the depth of 
(a)

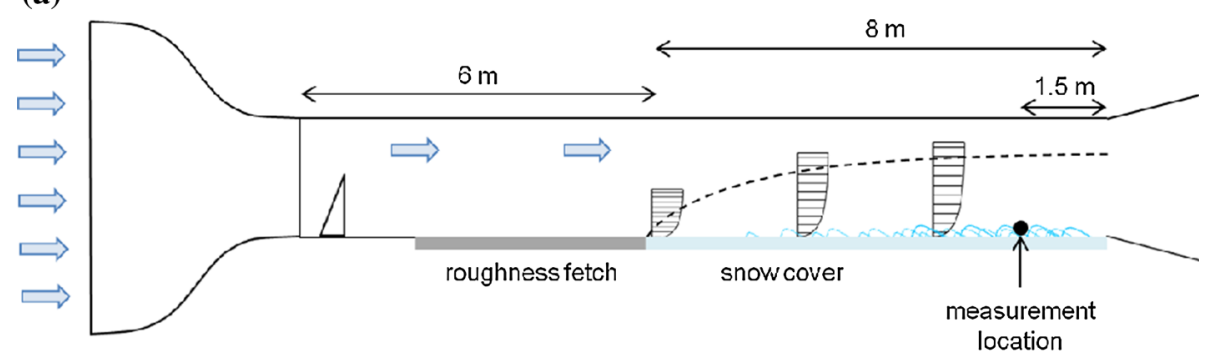

(b)

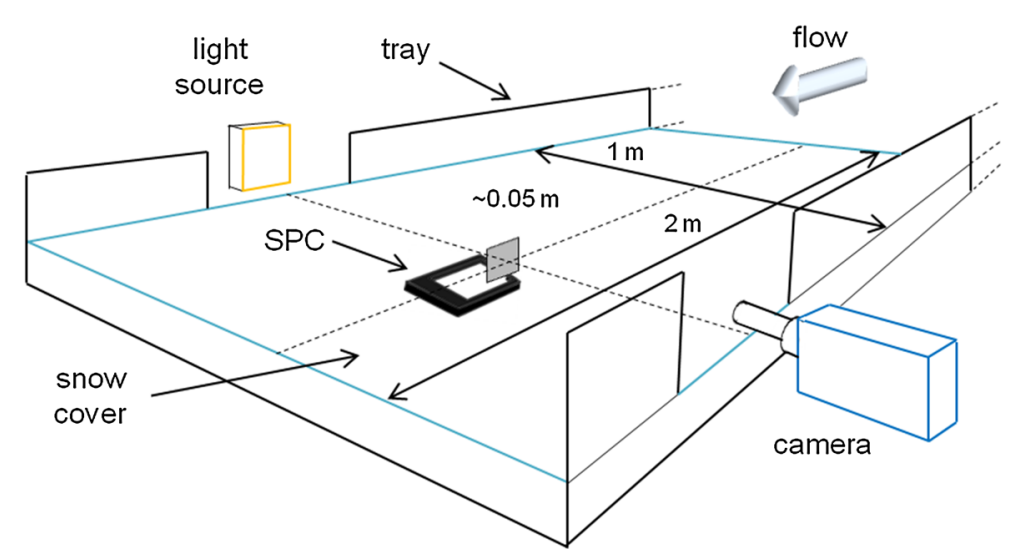

Fig. 1 Sketch of the wind tunnel (a), sketch of the PTV set-up in the wind tunnel (b), adapted after Paterna et al. (2016)

fresh snow on the trays reaches between $0.07 \mathrm{~m}$ and $0.15 \mathrm{~m}$, the trays are positioned into the wind tunnel with as little disturbance as possible. The floor in the wind tunnel can be vertically adjusted such that the depth of the snow cover matches the height of the floor and the roughness elements in the upwind end of the wind tunnel, as well as the light and the camera system in the measurement section at the downwind end of the tunnel. Previous experiments conducted in the same wind tunnel reported a boundary-layer depth of approximately 0.25 m (Clifton et al. 2006).

\subsection{Snow-Particle Counter}

The first snow-particle counter was introduced by Schmidt (1977) and the concept developed further by Kimura (1993) to the well-established version SPC-S7 (Niigata Denki Co.) Many studies using this technique were performed in the past (see e.g. Nishimura et al. 1998; Sugiura et al. 1998; Doorschot et al. 2004; Nishimura and Nemoto 2005; Clifton et al. 2006; Guala et al. 2008; Gordon and Taylor 2009; Gordon et al. 2009). The working principle is based on a focused light emitter and photodiode that detects the shadows of particles drifting through the control volume $(2 \mathrm{~mm} \times 25 \mathrm{~mm} \times 0.5 \mathrm{~mm})$. Based on the amplitude of the photodiode's voltage signal it is possible to classify the snow-particle diameter as one of 32 particle diameter bin classes between $0.04 \mathrm{~mm}$ and $0.5 \mathrm{~mm}$ (assuming that the particles are spherical). Detected particles with a diameter larger then $0.5 \mathrm{~mm}$ are assigned to the largest 
diameter bin, while their exact diameter remains undetermined. The raw signal is sampled at $40 \mathrm{kHz}$. Particle numbers with the corresponding diameter class are used to calculate the mass flux. For the three parameters, particle number, particle diameter, mass flux, the 1 -s averaged signal is delivered as an output based on the manufacturer's software. Before the experiments described herein the device was recalibrated by the manufacturer. For the experiments, the SPC was mounted on a traverse that allowed positioning in three dimensions in the wind-tunnel test section.

\subsection{Particle Tracking Velocimetry Based on Shadowgraphic Images}

The second measurement technique employed to determine the mass flux of drifting snow (or sand) is PTV based on shadowgraphic imaging. The shadowgraphic system consists of a light source (standard $500 \mathrm{~W}$ halogen) and a high speed CMOS camera $(1024$ pixel $\times 1024$ pixel) equipped with a telescopic lens (Nikon, focal length $=80-180 \mathrm{~mm}$ ). Unlike the pointwise measurement of the SPC, the PTV method is able to record series of two-dimensional images of drifting snow particles. Alongside the information about particle numbers, size distribution and mass flux, the PTV image analysis allows visual inspection of the processes in the saltation layer. This facilitates the understanding of processes in the lowest part of the boundary layer and enables a deeper analysis of the saltation processes (for a schematic drawing of the set-up, refer to Fig. 1b).

Camera and light source are positioned outside of the wind tunnel's sidewalls, perpendicular to the flow direction such that they add no disturbance to the flow. To allow vertical adjustment of the field-of-view, the camera is mounted upon a translation stage. A translucent paper and a Fresnel lens with a focal length of $35 \mathrm{~mm}$ are mounted in front of the light source to diffuse the light and parallelize the light rays. Prior to every experiment, the camera has to be focused and calibrated by means of a transparent ruler placed into the field-of-view. The image calibration and acquisition was managed with LaVisions's Davis 8.2 software. For all the experiments performed, the pixel length corresponds to approximately $50 \mu \mathrm{m}$, resulting in an approximate field-of-view of $50 \mathrm{~mm} \times 50 \mathrm{~mm}$. In order to obtain high speed images, the recorded images are initially saved on the random access memory of the camera and afterwards stored onto the hard disk. The random access memory allows recording up to 5456 images at the maximum resolution of 1024 pixels $\times 1024$ pixels.

The PTV set-up acquires images along a two-dimensional domain parallel to the flow direction. The third dimension is the depth of the domain, also referred as depth-of-field, where the particles are detected. The depth-of-field can be quantified since increasing distance from the focal plane causes particles to become increasingly blurred. The size of the depthof-field linearly decreases with decreasing particle size (for an illustration of this dependency, refer to Kim and Kim 1994, Fig. 7). The slope of this linear function depends on the depth of focus, which is limited by the camera settings (aperture, for all experiments on focal length $\mathrm{f} / 8$ ) as well as by the parameters in the post-processing. For the calculation of mass flux and volume flux, the depth-of-field was accurately calibrated by means of a target image with a matrix of dots with diameters between $50 \mu \mathrm{m}$ and $1 \mathrm{~mm}$. This target image was placed on a transparent plate in the middle of the field-of-view and translated towards the camera across the depth of focus with steps of $1 \mathrm{~mm}$ for a total of $60 \mathrm{~mm}$ centered on the depth of focus of the camera. Based on the recognition of the different particle sizes at different distances from the focal plane, the slope of the linear relation between particle diameter and depthof-field could be calculated. The processing of the depth-of-field calibration was performed according the instructions in the software manual (LaVision-GmbH 2011). 


\subsubsection{Post-Processing}

For the calculation of the velocity and the properties of the particles, a post-processing based on the recorded pictures was performed. The post-processing of the images identified the individual particles and their properties within each image (the settings of each parameter for the parametric study are explained in the following Sect. 2.3). The individual steps for the post-processing are as follows,

- Prior to the first segmentation, the raw images are inverted. For this purpose, the shadow images are subtracted from a reference image. The reference image is produced by a sliding maximum filter such that it represents the background image without particles. In the inverted image, the particles appear brighter than the background,

$$
\text { Inverted Image }=\text { Reference Image }- \text { Shadow Image }
$$

- Based on that inverted picture, a particle-recognition filter is applied. Applying this filter, a global threshold as a percentage between the maximum and the minimum light intensity within each image is defined. The algorithm attempts to find coherent areas above that threshold and defines them as particles (refer to Fig. 2a for the raw image and to Fig. 2b for the image after the particle-recognition filter).

- The second segmentation analyzes the previously recognized particle areas individually. Within this segmentation, two thresholds are defined for the particle-property filter. A high-level and a low-level threshold. All pixels intensities are compared to the maximum pixel intensity for the individual segment. The algorithm counts all pixels with intensities higher than the low-level threshold as one set, and all the pixels with intensity higher then the high-level threshold as another set. For each of the two sets, the area and the diameter

(a)

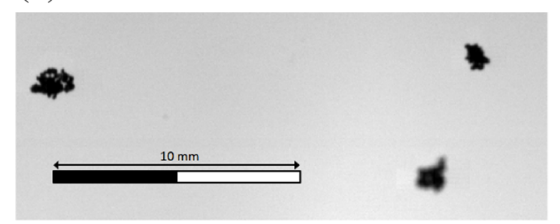

(b)

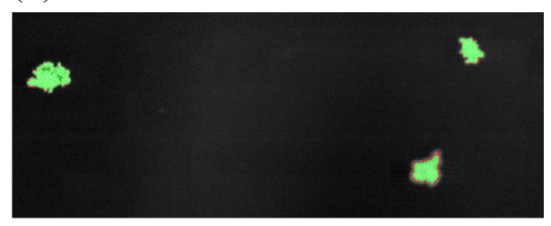

(c)

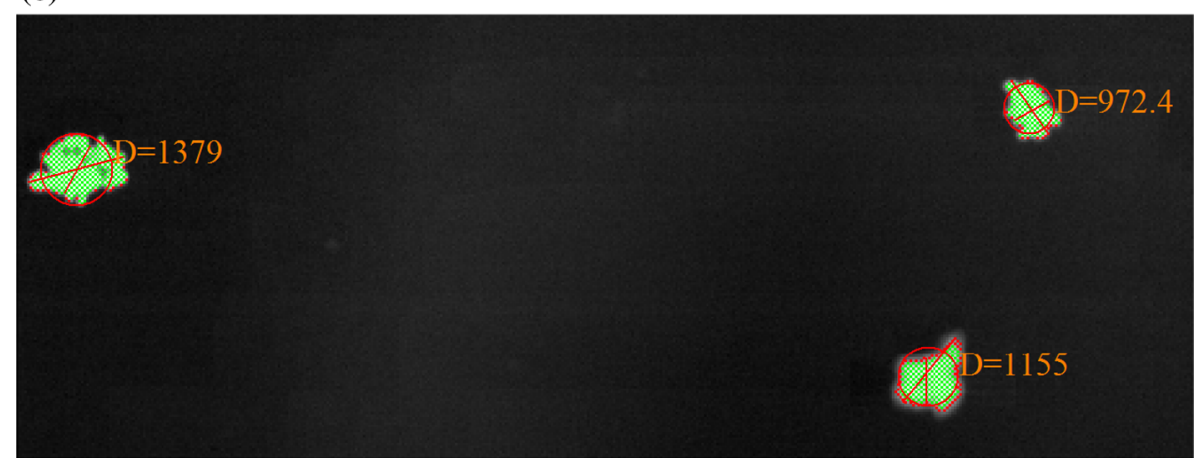

Fig. 2 Details of a captured raw image (a), and the inverted image after the particle-recognition filter (b). The green highlighted areas are recognized particles. The inverted image after the particle-property filter (c). The long (low-level) and short (high-level) axes are drawn in red as well as the diameter $(\mu \mathrm{m})$ in orange 


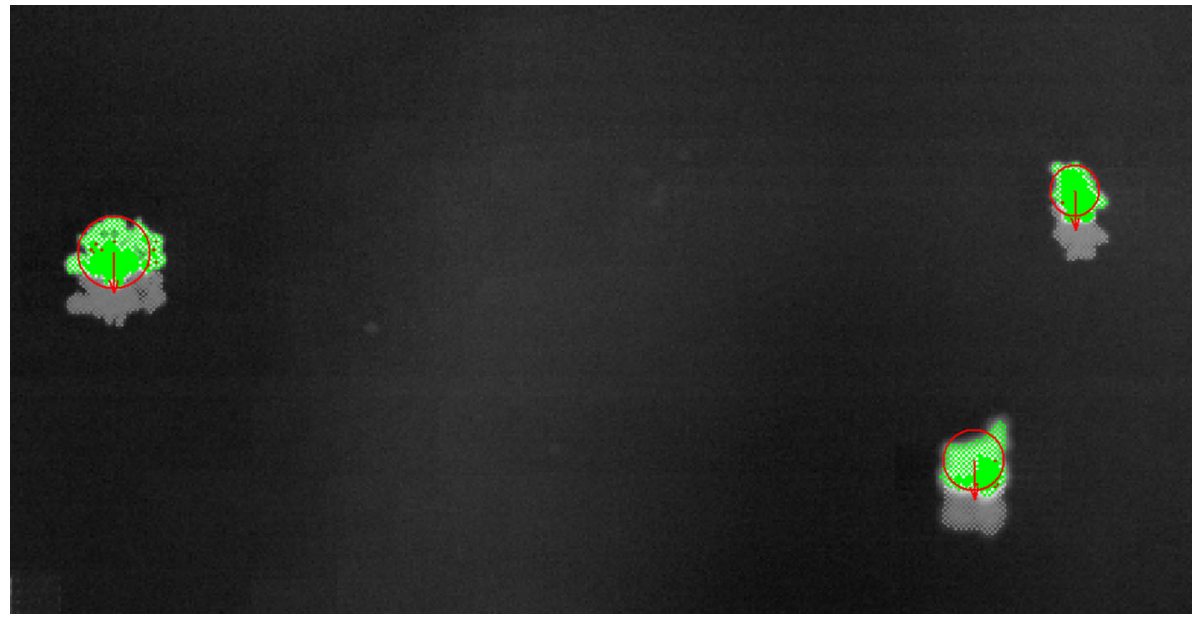

Fig. 3 The velocity calculation was performed based on the shift between two consecutive images. The green area represents the particle's initial position, the grey area is the final position

are calculated. The diameter and centre of the particle is then calculated as the average to the two sets (Fig. 2c). The calculated diameter is a measure of the particle size.

- The third filter that was applied is a particle filter. This filter sets a threshold for the maximal low-level area as a percentage of the high-level area. The filter is designed to exclude de-focused particles or structures that are recognized as particles, but do not represent suitable contributions because of their insufficiently clear detection.

- The next step in the post processing is the calculation of the particle velocity $v_{p_{i}}$, done by using a particle tracking algorithm. Before applying the velocity calculation, information about position and diameter are stored in two lists. To identify pairs of particles, a shift and an interrogation window are defined for each particle in two consecutive images. Based on the cross-correlation between the interrogation window of the images, matching particle pairs can be defined. To avoid a misinterpretation of particle pairs, a maximal shift and a maximal deviation between the diameters are defined. The velocity calculation is based on the separation time between two consecutive images; refer to Fig. 3 for an illustration of the particles velocity.

- An additional border correction is applied for the computing of the particle detection area. The first segmentation just recognizes particles that are entirely within the defined image area. Particles that are just partially within the field-of-view are not considered. Therefore, the image border corresponding to half of the particle diameter $d_{i}$ does not contribute to the image detection area. The field-of-view (FOV) for the volume calculation was cropped for each particle individually based on its diameter,

$$
\begin{aligned}
& F O V_{x, i}=F O V_{x}-d_{i}, \\
& F O V_{y, i}=F O V_{y}-d_{i} .
\end{aligned}
$$

- According to the previously described slope of the depth-of-field (DOF) $m_{D O F}$, the depth-of-field for each particle could be calculated based on its diameter,

$$
D O F_{i}=m_{D O F} \cdot d_{i}
$$


Together with the density of the snow particles $\rho_{\text {snow }}$, the particle velocity and the fieldof-view, the mass flux for each particle individually is then given by

$$
q_{i}=\rho_{\text {snow }}\left(\frac{d_{i}^{3}}{F O V_{x i} \cdot F O V_{y_{i}} \cdot D O F_{i}}\right) v_{p_{i}} .
$$

The mass flux for each timestep is calculated as the sum of the mass flux of each particle within the image,

$$
Q(t)=\sum_{i} q_{i}
$$

The particle tracking algorithm is part of the software package Davis provided by LaVision, $\mathrm{GmbH}$. For this study, the Davis software was used for the particle recognition, property calculations, filtering and velocity calculations. The reference values to start the parametric study were taken from the software's manual (LaVision-GmbH 2011). The calculation of the mass flux including the border correction and the depth-of-field estimation was done with MATLAB software. For further details on the Davis software refer to the mentioned manual provided by LaVision-GmbH (2011).

\subsection{Experiments}

In order to correlate particle numbers, size distribution and mass flux measured by both techniques, two different tests were performed. The first test was designed as a calibration study in rather controlled conditions, the second test was a typical drifting snow test with the aim of testing the measurement techniques in more complex and realistic conditions. For both tests, the PTV and SPC techniques were compared for different parameter settings on the PTV image analysis side in order to determine the most suitable processing parameters for the shadowgraphic image processing. Parameters that were addressed for the processing of the PTV images were the thresholds for particle recognition, for the particle property (high-level-low-level), the particle filter and field-of-view. The percentages for the variation of the threshold as used in the parametric study can be found in Table 1. The theory to these parameters is explained in the previous Sect. 2.2.1.

The size of the field-of-view was cropped in both directions, horizontally and vertically. Cropping of the field-of-view was introduced to investigate the dependency of the result on the domain size. For the variation of the field-of-view, the values of the other parameters were set according to the previously determined best values.

Table 1 Parameter thresholds settings for the parametric study in percentages (Particle recognition (PR), Particle property (PP: low-level-high-level), Particle filter (PF), domain size of the field-of-view horizontally (dFOVx), domain size of the field-of-view vertically (dFOVy)

\begin{tabular}{lllllll}
\hline PR & 20 & 30 & 40 & 50 & 60 & 70 \\
PP & $25-75$ & $30-70$ & $35-65$ & $40-60$ & $45-55$ & $50-50$ \\
PF & 125 & 150 & 175 & 200 & 225 & 250 \\
dFOVx & 100 & 43 & 32 & 21 & 11 & \\
dFOVy & 100 & 36 & 27 & 18 & 9 & \\
\hline
\end{tabular}


Fig. 4 Images from the calibration study with artificially scattered snow particles and the $\mathrm{SPC}$ visible in the image



\subsubsection{Calibration Experiment}

The first set of experiments was designed such that the SPC is visible in the PTV images and preferably measuring the same particles (Fig. 4). Snow particles were artificially scattered using a sieve. The driving force for particle movement was gravity only, resulting in relatively low particle velocities and a wide, homogeneous distribution of particles. The PTV recordings were conducted with an exposure time $t_{\exp }$ of $0.33 \times 10^{-5} \mathrm{~s}$. According to Bagnold (1941), the selection of the exposure time should be based on maximal expected velocity $U_{\max }$ of the minimal expected particle diameter $d_{\min }$,

$$
t_{\exp }=\frac{d_{\min }}{U_{\max }}
$$

In the case of the calibration experiment, the minimal diameter according the SPC bin was $d_{\min }=0.04 \mathrm{~mm}$, the maximal velocity was $U_{\max } \approx 1 \mathrm{~m} \mathrm{~s}^{-1}$, resulting in a maximal $t_{\exp }=0.4 \times 10^{-5} \mathrm{~s}$. With $0.33 \times 10^{-5} \mathrm{~s}$, we ensured capturing sharp enough images. For each sample, a pair of images was taken, and based on the particle path from the first to the second image of this pair, the particle velocity could be calculated. The frequency to record this pair of images is called the separation time. For the calibration experiment, the separation time was set to $0.001 \mathrm{~s}$ (frequency for the separation time 1 $\mathrm{kHz}$ ). The sampling frequency was set to $50 \mathrm{~Hz}$ guaranteeing the detection of all particles falling through the sensor. In total, three different tests were processed (experiments $\mathrm{C} 1-$ $\mathrm{C} 3$ ), and during the calibration experiments, the air temperature varied between -2.6 and $-3.4^{\circ} \mathrm{C}$.

Besides the calibration experiment with snow, an additional experiment with the same setup and quartz sand particles was performed. The sand particles had a particle-size distribution given by the manufacturer from 0.1 to $0.6 \mathrm{~mm}$ in diameter. This test was performed in order to compare the complex-shaped snow particles to more mono-dispersed and round particles. 

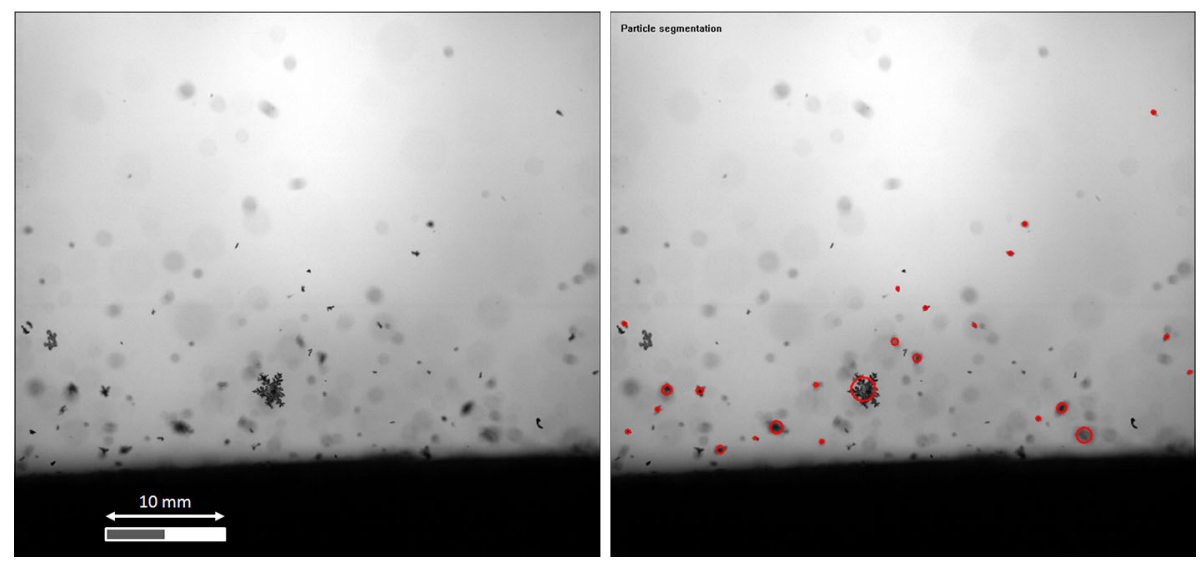

Fig. 5 On the left, a raw shadowgraphic picture of snow in saltation, and on the right, the same picture after processing with particle-recognition, particle-property and particle-filter methods. The red circles in the right picture indicate the calculated particle diameter

\subsubsection{Drifting Experiment}

The second experiment was a standard drifting test on a natural snow cover with the SPC placed a few millimetres downstream of the PTV system's field-of-view with the middle of the SPC measurement volume aligned to the middle of the field-of-view (Fig. 5). The wind speed was set high enough to allow sufficient saltation, with the wind speed in the freestream region measured with a propeller-type anemometer (MiniAir 20, Schiltknecht Messtechnik AG).

Previous publications report an exponential decrease of the horizontal mass flux with height (Sugiura et al. 1998; Nishimura and Nemoto 2005; Guala et al. 2008). As a result, the most significant portion of the mass is transported near the surface. Hence the fieldof-view was focused for the detection of the inner saltation layer, where the peak of the mass flux occurs. Focusing on a small region of the saltation layer allows us to increase the spatial resolution of the acquired images in order to better detect the smallest particles. As with the calibration tests, three drifting tests were conducted (Table 2). The separation time between the two images of each pair was set to $2.78 \times 10^{-4} \mathrm{~s}(3.6 \mathrm{kHz})$, the image pairs were sampled at $20 \mathrm{~Hz}$, which resulted in an experimental duration of $136.4 \mathrm{~s}$. Previous experience, as well as Bagnold's relation for the exposure time (Eq. 5), suggested using an exposure time short enough in order to freeze the particle motions and thus avoid blurring. The calibration experiment proved that the smallest recognizable particles with the current set-up were $d_{\min } \approx 0.1 \mathrm{~mm}$. With the freestream velocity set at $U_{\text {free }}<11.8 \mathrm{~m} \mathrm{~s}^{-1}, t_{\mathrm{exp}}$ should have been set to $8.475 \times 10^{-6} \mathrm{~s}(118 \mathrm{kHz})$ (particles near the surface are generally slower),

Table 2 Conditions and settings of the drifting experiment

\begin{tabular}{lllllll}
\hline & $U_{\text {free }}\left(\mathrm{m} \mathrm{s}^{-1}\right)$ & $T_{\text {air }}\left({ }^{\circ} \mathrm{C}\right)$ & $\begin{array}{l}\text { Number of } \\
\text { images }\end{array}$ & $\begin{array}{l}\text { Exposure } \\
\text { times }\end{array}$ & $\begin{array}{l}\text { Separation } \\
\text { time }(\mathrm{kHz})\end{array}$ & $\begin{array}{l}\text { Sampling } \\
\text { frequency }(\mathrm{Hz})\end{array}$ \\
\hline $\mathrm{D} 1$ & 14 & $-1.2 \pm 0.1$ & 2728 & $1.22 \times 10^{-5}$ & 3.6 & 10 \\
$\mathrm{D} 2$ & 10.7 & $-1.3 \pm 0.1$ & 2728 & $1.22 \times 10^{-5}$ & 3.6 & 20 \\
$\mathrm{D} 3$ & 11.8 & $-1.3 \pm 0.1$ & 2728 & $1.22 \times 10^{-5}$ & 3.6 & 20 \\
\hline
\end{tabular}




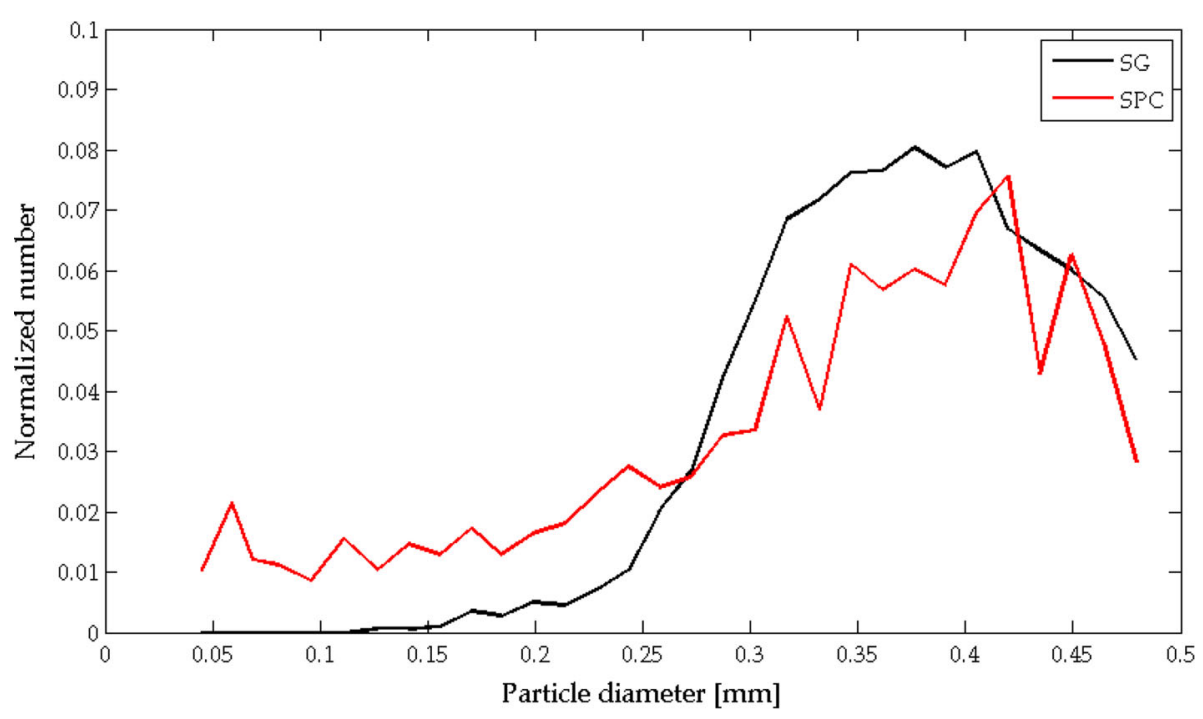

Fig. 6 Particle-size distribution of the calibration experiment with sand particles. The vertical axis shows the normalized number of particles

resulting in underexposed images. Therefore, for all the three experiments, the exposure time was set to $1.22 \times 10^{-5} \mathrm{~s}(82 \mathrm{kHz})$ in order to have a good compromise between light intensity, contrast and exposure time.

One shortcoming of both methods is the assumption of spherical particles for the calculation of the mass flux. Especially for larger particles, when particles are shaped as dendrite (refer to Fig. 5), the error in the absolute values of the mass flux may be considerable. For the current study, this assumption should not weaken the correlation, since it is valid for both techniques.

\section{Results}

\subsection{Calibration Experiment}

We first present a representative sand experiment (refer to Sect. 2.3), which we conducted in preparation for the more complex snow experiments. Mass calculations based on the assumption of spherical grains are less uncertain than for the highly irregular snow-particle shapes. Figure 6 shows the particle-size distribution of this sand experiment, and confirming that both instruments interpret the particle size similarly. The particle numbers are normalized by dividing the numbers of the individual classes by the total number of particles detected by the corresponding technique. In contrast to the calibration experiment with snow, the sand could not be sieved homogeneously over the whole field-of-view, the requirements for a calculation of the mass flux with PTV image analysis were not fulfilled and therefore no mass-flux calculation for the sand is presented here.

\subsubsection{Mass Flux and Particle-Size Distribution}

The two time series of the mass flux recorded by the SPC and the PTV method were correlated using MATLAB and the Spearman-correlation function. The time series from the 
shadowgraphic PTV were down-sampled to match the sampling frequency of the SPC. Since the range of the particle diameter on the SPC side is bounded between $0.04 \mathrm{~mm}$ to $0.5 \mathrm{~mm}$ (larger particles are assigned to the 0.5 -mm-diameter class), particles on the PTV side with a diameter $>0.5 \mathrm{~mm}$ are also counted as $0.5 \mathrm{~mm}$. This restriction on the PTV side was intended to reduce potential errors and disagreement between the two methods. Figure 7a shows the mass-flux signal for experiment $\mathrm{C} 1$. The average particle speed recorded by the PTV system for the calibration experiments was between $0.96 \mathrm{~m} \mathrm{~s}^{-1}$ and $1.13 \mathrm{~m} \mathrm{~s}^{-1}$ (experiment $\mathrm{C} 1,1.11 \mathrm{~m} \mathrm{~s}^{-1} ; \mathrm{C} 2,0.96 \mathrm{~m} \mathrm{~s}^{-1}$; and C3 $1.13 \mathrm{~m} \mathrm{~s}^{-1}$ ). Already here, a satisfying agreement between the signals of the mass flux for SPC and PTV is obtainable, except a slight discrepancy for the events with high mass flux. Figure $7 \mathrm{~b}$ shows the normalized particle-size distribution for the same experiment.

It can be noted that for this experiment, the PTV records many more particles compared to the SPC, and can be explained by the larger measurement volume of the PTV images. Furthermore, it is noticeable in the particle-size distribution in Fig. 7b that the PTV method fails to detect particles in the range $0.04-0.15 \mathrm{~mm}$.

\subsubsection{PTV Parameter Sensitivity}

Figure 8 shows the results of the parametric study of the PTV post-processing, with the ratio of the PTV and SPC mass flux and their correlation coefficient. On the horizontal axis of each panel, the thresholds in percentage for the three processing parameters PR, PP and PF (refer to Sect. 2.2.1) are listed. Overall the correlation as well as the ratio of the mass flux were very satisfying for all cases considered. As previously described, the filter for the particle recognition influences how many particles are recognized. For low particle-recognition thresholds, the ratio of the total mass flux increases (Fig. 8a). This can be explained by considering that with a low threshold for the particle recognition, more pixels in the image are interpreted as particles resulting in an increase of the mass flux. This effect seems to be linear, since it does not affect the correlation between the two signals significantly. For the particle-recognition filter, the correlation was always between 0.91 and 0.96 , the ratio of the mass flux between 1.15 and 0.85 . The highest correlation overall was found in the range of $40-50 \%$. The ratio of the mass flux for a particle-recognition threshold of $50 \%$ is slightly closer to one than that for $40 \%$. Except for the results for $\mathrm{C} 3$, the results for the tests on particle recognition are very similar to each other. Also the results for the three tests with different particle-property thresholds are very similar to each other, for both correlation and ratio of the mass flux. Small particle-property thresholds, resulting in a large difference between low-level and high-level intensity, reduce the ratio of the mass flux (Fig. $8 \mathrm{c})$. This behaviour can be explained by the fact that a large difference between low-level and high-level intensity leads to a unfavourable ratio for the consecutive particle-filter threshold. Hence many particles are removed by the particle filter. Similar to the particle-recognition threshold, this effect seems to be linear as well and therefore is not weakening the correlation strongly. Compared to the particle-recognition threshold, the ratio of the mass flux shows a clearer optimum for all experiments, which is at a particle-property threshold for the low-level and high-level pixel intensity of 40-60\%. Also the variation of the particle-filter threshold demonstrated good correlation, as well as similar effects on the mass flux ratio. As described previously, for low particle-filter thresholds, many particles do not satisfy the threshold and are therefore excluded from processing. This only affects the ratio of the mass flux and not the correlation between the two time series. The best correlation and mass-flux ratio for the particle-filter threshold is $175 \%$ (mean correlation coefficient 0.934 , ratio of the mass flux is 0.94$)$. 

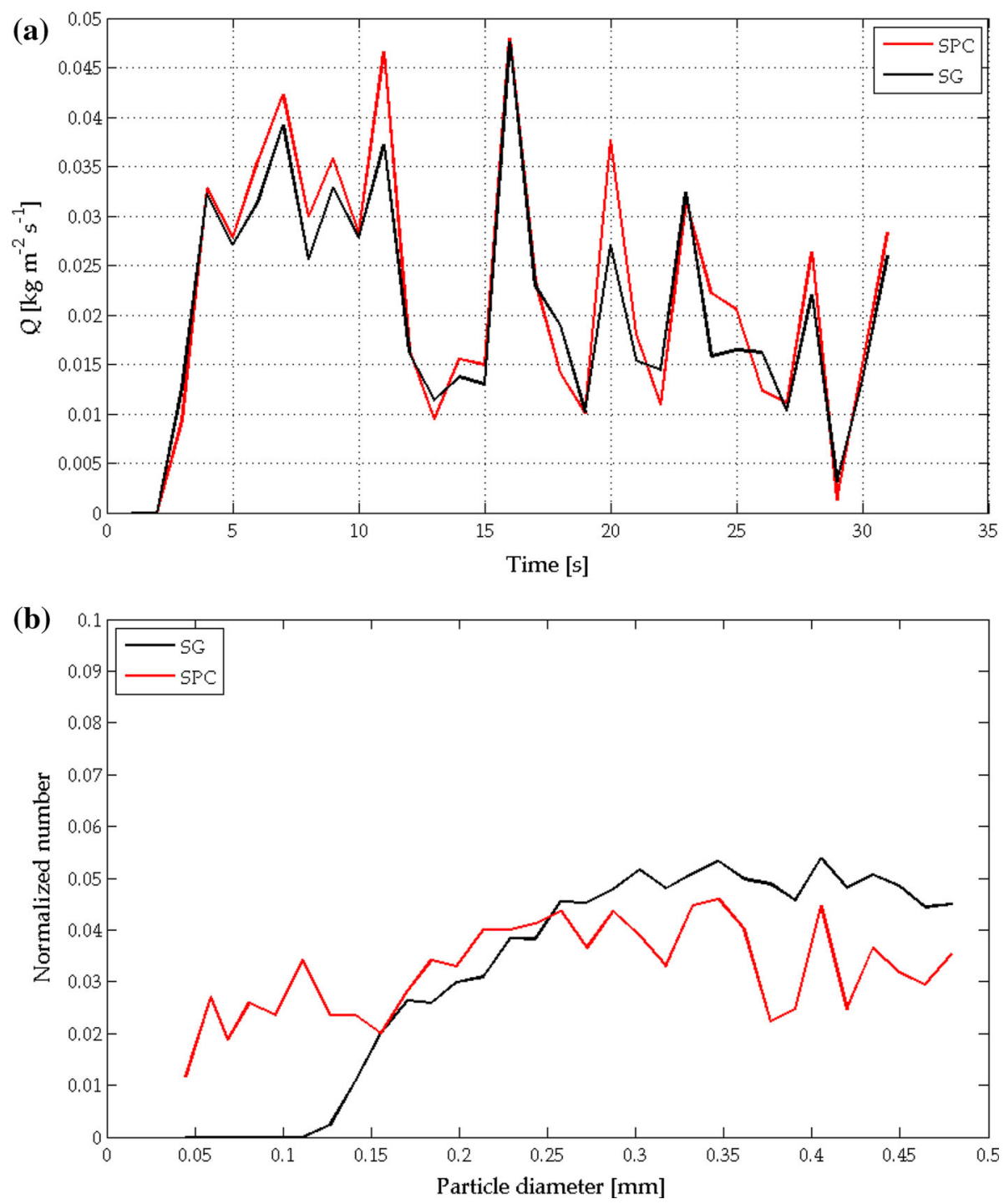

Fig. 7 Results of experiment C1-mass flux signal presented as 1-s averages (a), particle-size distribution with the vertical axis showing the normalized number of particles (b). Correlation $r=0.954$, total mass flux ratio $Q_{P T V} / Q_{S P C}=0.937$, total number of particles $\mathrm{PTV}=13,265$, total number of particles $\mathrm{SPC}=1208$. PTV settings were chosen according to the best case settings for the calibration experiment $(P R=50 \%, P P$ $=40-60 \%, P F=175 \%, d F O V x=d F O V y=100 \%)$

In the parametric study discussed above, the ratio of the mass flux and the correlation coefficient results from varying only one parameter at a time, while the other parameters were kept constant. For the sake of completeness a parametric study was also conducted by varying two parameters at a time, validating that the results discussed for the individual parameters are not biased by the choice of the other parameters. The ratios of the mass flux and correlation coefficients for the variations of two different parameters at a time are shown in Fig. 9. The two-parameter study was conducted based on the data of $\mathrm{C} 1$. The best fit 
(a)

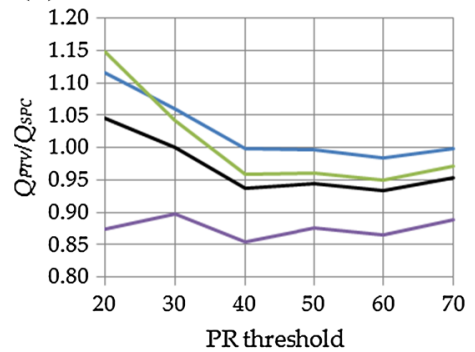

(c)
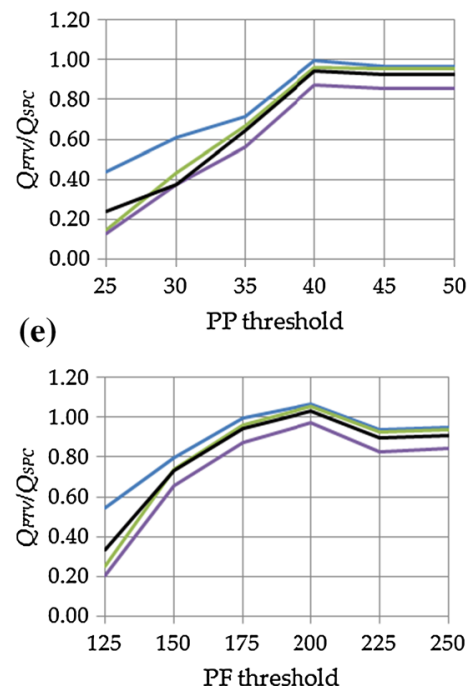

(b)

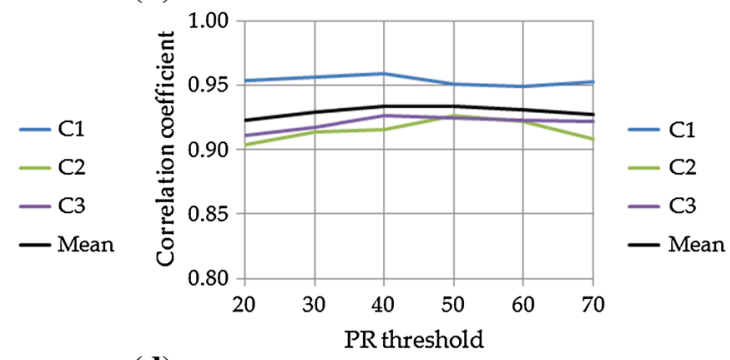

(d)

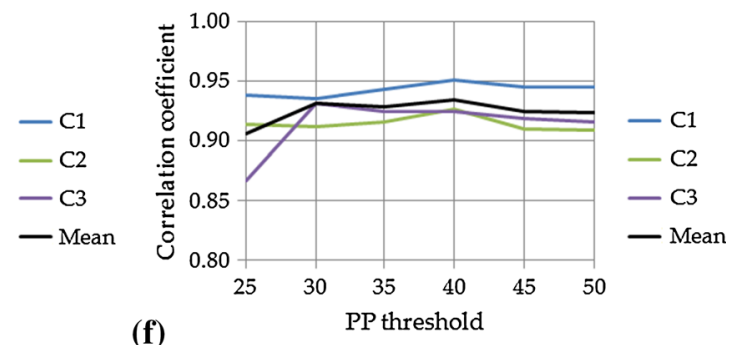

(f)

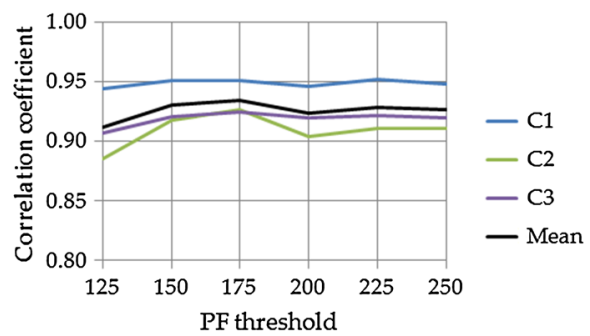

Fig. 8 Results of the parametric study for the calibration experiment. On the left side, the results of the ratio of the PTV and the SPC mass flux $\left(Q_{P T V} / Q_{S P C}\right)$ is shown for the different thresholds: $P R(\mathbf{a}), P P(\mathbf{c}), P F$ (e). On the right, the results of the correlation coefficient for the mass-flux time series are presented for the different thresholds: $P R(\mathbf{b}), P P(\mathbf{d}), P F(\mathbf{f})$

parameters from the results presented previously with the variation of one single parameter at a time could be confirmed. In the case of the particle-recognition threshold, the ratio of the mass flux and the correlation coefficient are optimized for a particle recognition around $50 \%$ and ratio and correlation are minimally affected by the choice of the particle-property or particle-filter thresholds. Furthermore, the choice of the particle-property threshold was confirmed to be optimized at a low-level of $40 \%$ and a high-level of $60 \%$ even when varying the particle-filter threshold at the same time.

Figure 10 shows the results for the ratio of the mass flux and correlation coefficients due to the variation of the field-of-view. For the evaluation of the dependency of the domain size, the height/width of the field-of-view was normalized with the path length of a particle within a timestep in streamwise direction or with the height/width of the SPC in the direction perpendicular to the streamwise direction. The path of a particle $p$ is calculated as the mean particle velocity $u_{P T V}$ and the sampling frequency $f_{s}$,

$$
p=\frac{u_{\mathrm{PTV}}}{f_{\mathrm{s}}} .
$$


(a)

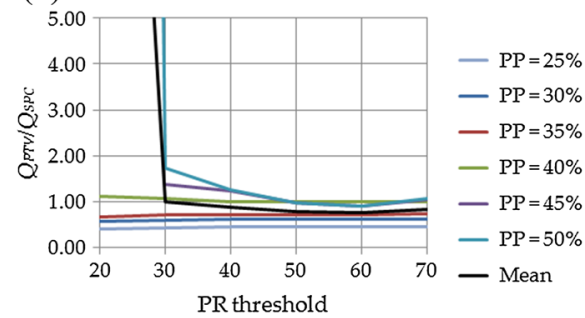

(c)

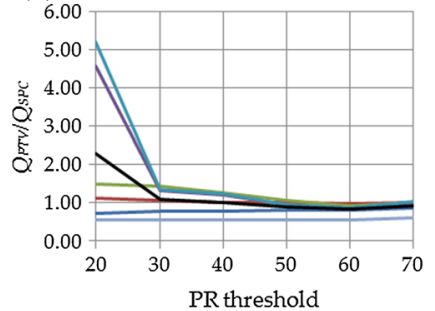

(e)

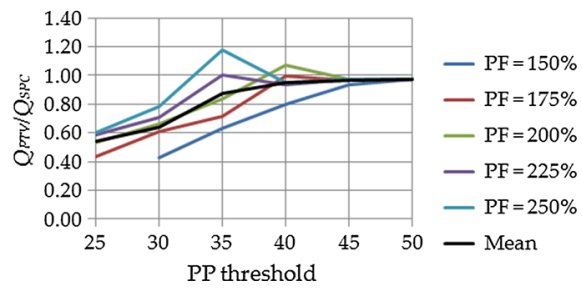

(b)



(d)

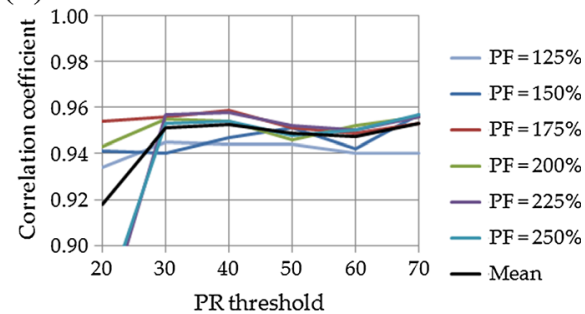

(f)

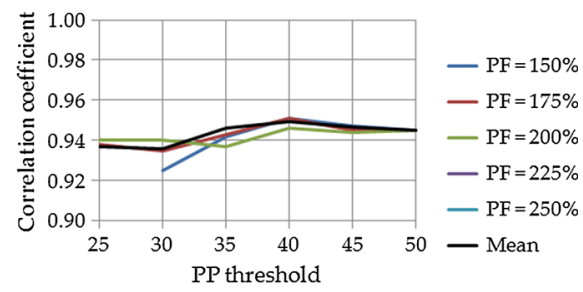

Fig. 9 Results of the parametric study for the calibration experiment. The horizontal axis represents the first parameter, the different colored lines represent the choice of the second parameter. On the left side, the mass flux ratio $\left(Q_{P T V} / Q_{S P C}\right)$ is shown for the combination of two different thresholds: $P R / P P(\mathbf{a}), P R / P F(\mathbf{c})$, $P P / P F(\mathbf{e})$. On the right, the correlation coefficient for the mass-flux time series are presented as a function of different thresholds: $P R / P P(\mathbf{b}), P R / P F(\mathbf{d}), P P / P F(\mathbf{f})$

The ratio of the mass flux and the correlation reaches a plateau as soon as the dimension of the field-of-view in the streamwise direction is approximately as long as the path of a particle between two consecutive images (i.e. the normalized length 1).

\subsection{Drifting Snow Experiment}

The analysis of the drifting snow tests followed the same scheme as seen for the calibration tests discussed above. In comparison to the calibration experiments, the length of the fieldof-view was now varied along the streamwise direction and the resulting mass flux proved to be more intermittent than in the previous experiment (refer to Fig. 11). Figure 12a shows the mass-flux time series for experiment D1, Fig. 12b shows the diameter distribution for the same experiment. In contrast to the calibration test, where the SPC detected more than 10 times less particles then the PTV, in the drifting experiments, the numbers of detected particles by the SPC were in a similar range. This large difference is mainly explained by the fact that in the case of the calibration experiment, where the particle sizes are more uniform, the PTV measurement volume is much larger than the SPC volume. In the case of the drifting experiment, due to the difference in detected particle sizes, the size of the 
(a)

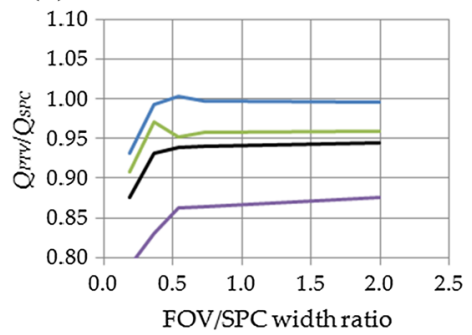

(c)

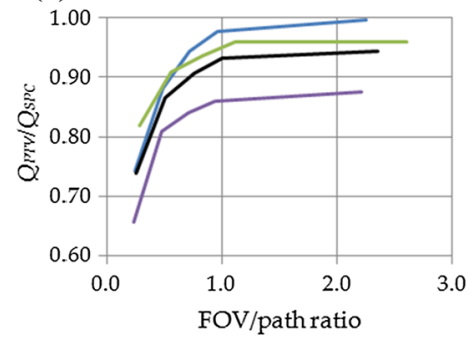

(b)
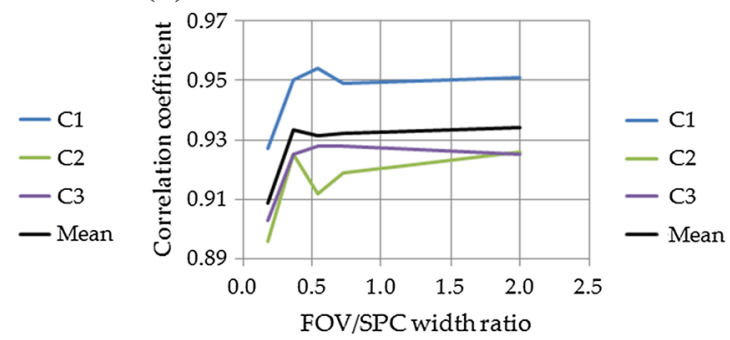

(d)

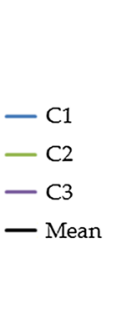

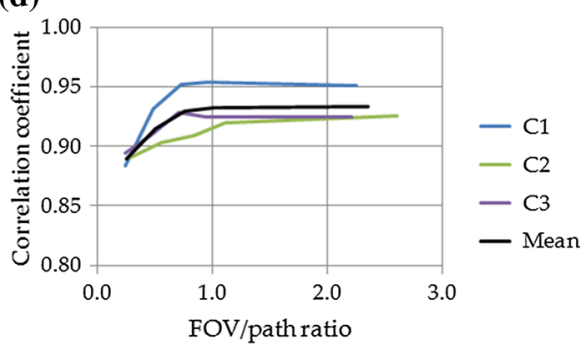

Fig. 10 Results of the parametric study for the calibration experiment. On the left side, the mass-flux ratio $\left(Q_{P T V} / Q_{S P C}, \mathrm{FOV} / \mathrm{SPC}\right.$ width ratio $(\mathbf{a}), \mathrm{FOV} /$ path ratio $\left.(\mathbf{c})\right)$ is shown for the different dimensions of the field-of-view. On the right, the correlation coefficient for the mass-flux time series are presented (FOV/SPC width ratio $(\mathbf{b})$, FOV/path ratio $(\mathbf{d}))$

Fig. 11 Shadowgraphic picture of snow in saltation during a drifting experiment

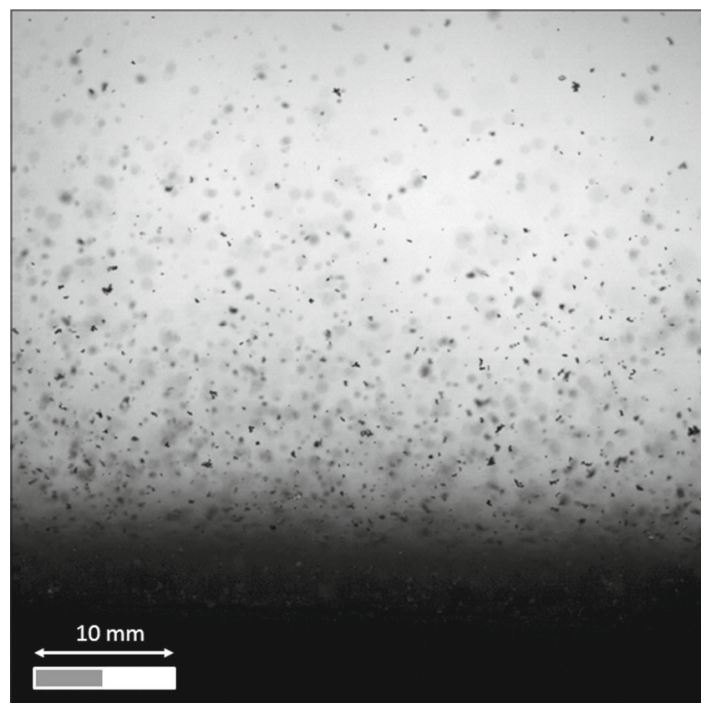

measurement volume is compensated by the SPC's capability to record smaller particles. In the experiments D1 and D3, the SPC recorded even more particles then the PTV system. Also the average particle velocity in the field-of-view recorded by the PTV was much higher (experiment D1, $6.9 \mathrm{~m} \mathrm{~s}^{-1}$; D2, $5.1 \mathrm{~m} \mathrm{~s}^{-1}$; and D3, $6.0 \mathrm{~m} \mathrm{~s}^{-1}$ ). A more detailed discussion of the particle numbers and diameter distribution is presented in the subsequent section. 



Fig. 12 Results of experiment D1-mass-flux signal presented as 1-s averages (a), particle-size distribution with the vertical axis showing the normalized number of particles $(\mathbf{b}) . r=0.83, Q_{P T V} / Q_{S P C}=1.016$, Total number of particles PTV $=12953$, total number of particles $\mathrm{SPC}=15,161$, PTV settings: $P R=50 \%, P P=$ $45-55 \%, P F=200 \%, d F O V x=d F O V y=100 \%$

The results for the correlation of the experiments with drifting snow are shown in Fig. 13 $\mathrm{b}, \mathrm{d}, \mathrm{f}$. Compared to the calibration experiments, the correlation between the two signals is slightly weaker. The results for optimal thresholds for particle recognition (50\%), particle property (45-55\%) and particle filter $(200 \%)$ are close to the results from the calibration study. While the best choice of the particle-recognition threshold is identical, the particleproperty and particle-filter thresholds are slightly higher compared to the calibration study. The parametric study for the drifting experiments reveals that the results from the particlerecognition threshold is the most difficult to judge as the range of optimal values is rather large. 
(a)

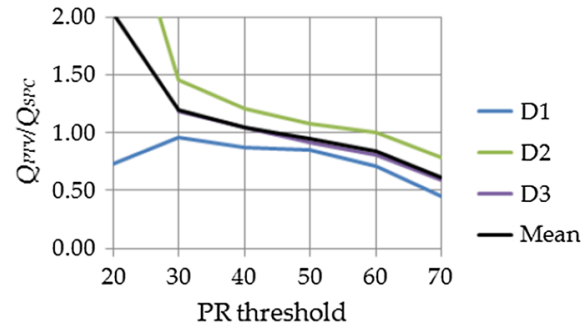

(c)

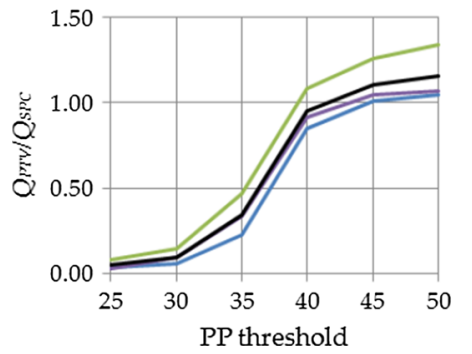

(e)

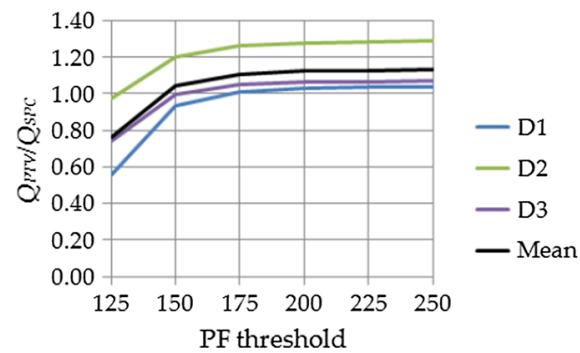

(b)

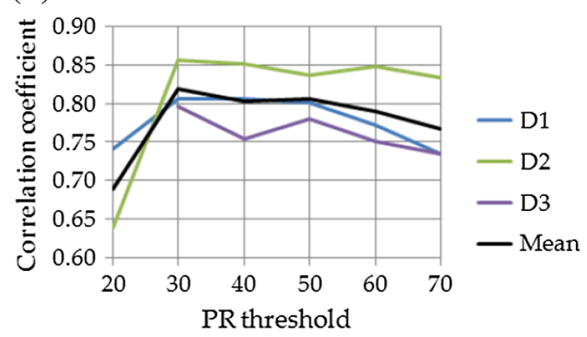

(d)



(f)

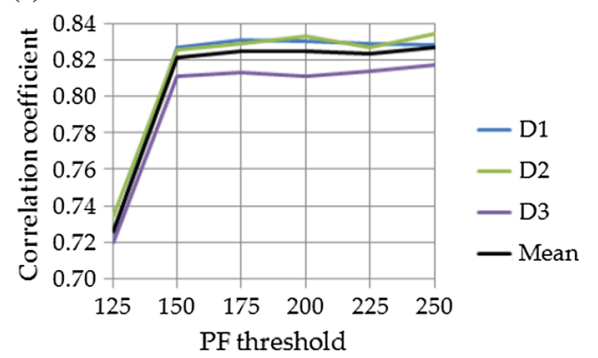

Fig. 13 Results of the parametric study for the drift experiment. On the left side, the mass flux ratio $Q_{P T V} / Q_{S P C}$ is shown for the different thresholds: $P R(\mathbf{a}), P P(\mathbf{c}), P F(\mathbf{e})$. On the right, the correlation coefficient for the mass flux time series are presented: $P R(\mathbf{b}), P P(\mathbf{d}), P F(\mathbf{f})$

The results from the correlation and the ratio of the mass flux suggest $50 \%$ as the optimal choice, even though a threshold set at $40 \%$ would not produce any significant difference in the results as well.

The results for the cropped image of the drifting snow experiment again show the sensitivity of the results to the field-of-view dimensions (refer to Fig. 14). For the streamwise direction, the correlation curve has a logarithmic shape. Compared to the calibration test, due to the greater velocity of the snow particles, as well as the trade off between sampling frequency and the length of the time series, the ratio between the field-of-view and path length is smaller. In order to reach a similar ratio like in the calibration experiment, the sampling frequency should have been set 10 times larger. Due to the limited camera memory, this would have lead to a time series duration of less then $30 \mathrm{~s}$. Considering the relevant time scales occurring in drifting snow events such an acquisition frequency would have limited the convergence of the statistics. As a consequence, the sampling frequency was reduced. Despite this reduction in the sampling frequency, the ratio of the mass flux recorded by the PTV and the SPC equals 1 at a ratio of the field-of-view to the particle path of approximately 
(a)

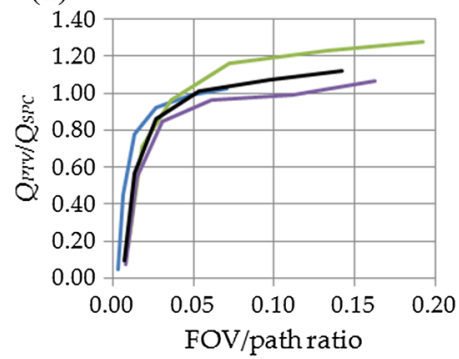

(c)

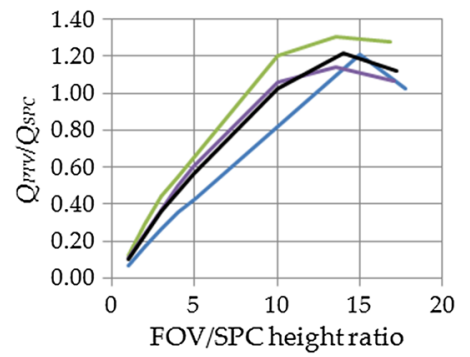

(b)

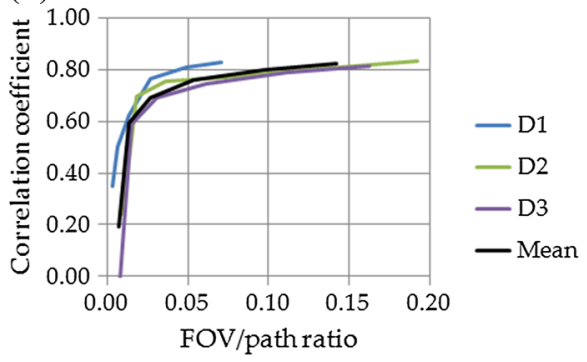

(d)

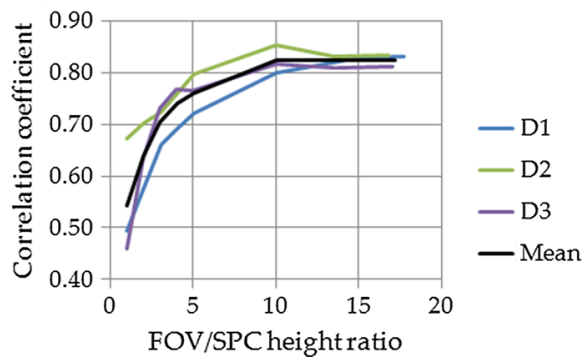

Fig. 14 Results of the parametric study for the drift experiment. On the left side, the mass flux ratio $Q_{P T V} / Q_{S P C}$ is shown for the different dimensions of the field-of-view (FOV/path ratio (a), FOV/SPC height ratio (c))). On the right, the correlation coefficient for the mass flux time series are presented FOV/path ratio (b), FOV/SPC height ratio $(\mathbf{d}))$

0.05. The correlation at that point is already at more than 0.75 and tends to increase further with increasing horizontal length of the field-of-view, although the PTV overestimates the mass flux compared to the SPC. Comparing this behaviour to the analogous plot from the calibration experiment, it can be assumed that the correlation would increase further along the $x$-axis. In the case of the vertical field-of-view, the best correlation and ratio of the mass flux is obtained when the field-of-view is 10 times larger then the height of the SPC (i.e. approximately $20 \mathrm{~mm}$ ). For the drifting experiment, no additional two-parameter study was conducted.

\subsection{Number of Particles}

Although the mass-flux signals from the SPC and PTV show a strong correlation, the particlesize distribution are significantly different between the two measurement techniques. Since the calculation of the mass flux for both techniques is based on the number of particles detected and their corresponding diameter estimation, we also correlated the timeseries of the number of particles from the two techniques. Therefore the particle number fluxes resulting from the optimal choice of the three different particle thresholds (particle recognition, particle property, particle filter) were analyzed. Figure 15a shows the number flux presented as 1$\mathrm{s}$ average for the calibration experiment, Figure $15 \mathrm{~b}$ shows the number flux of the drifting snow experiment. The correlation for the particle numbers in the calibration experiment were slightly larger for all three experiments $(r=0.931-0.965)$ compared to the correlation of the mass flux. Although for the calibration experiment, more than ten times less particles were recorded with the SPC than with the PTV (refer to Fig. 15a, $n p_{P T V} / n p_{S P C}=10.981$ ), the correlation was very high. 

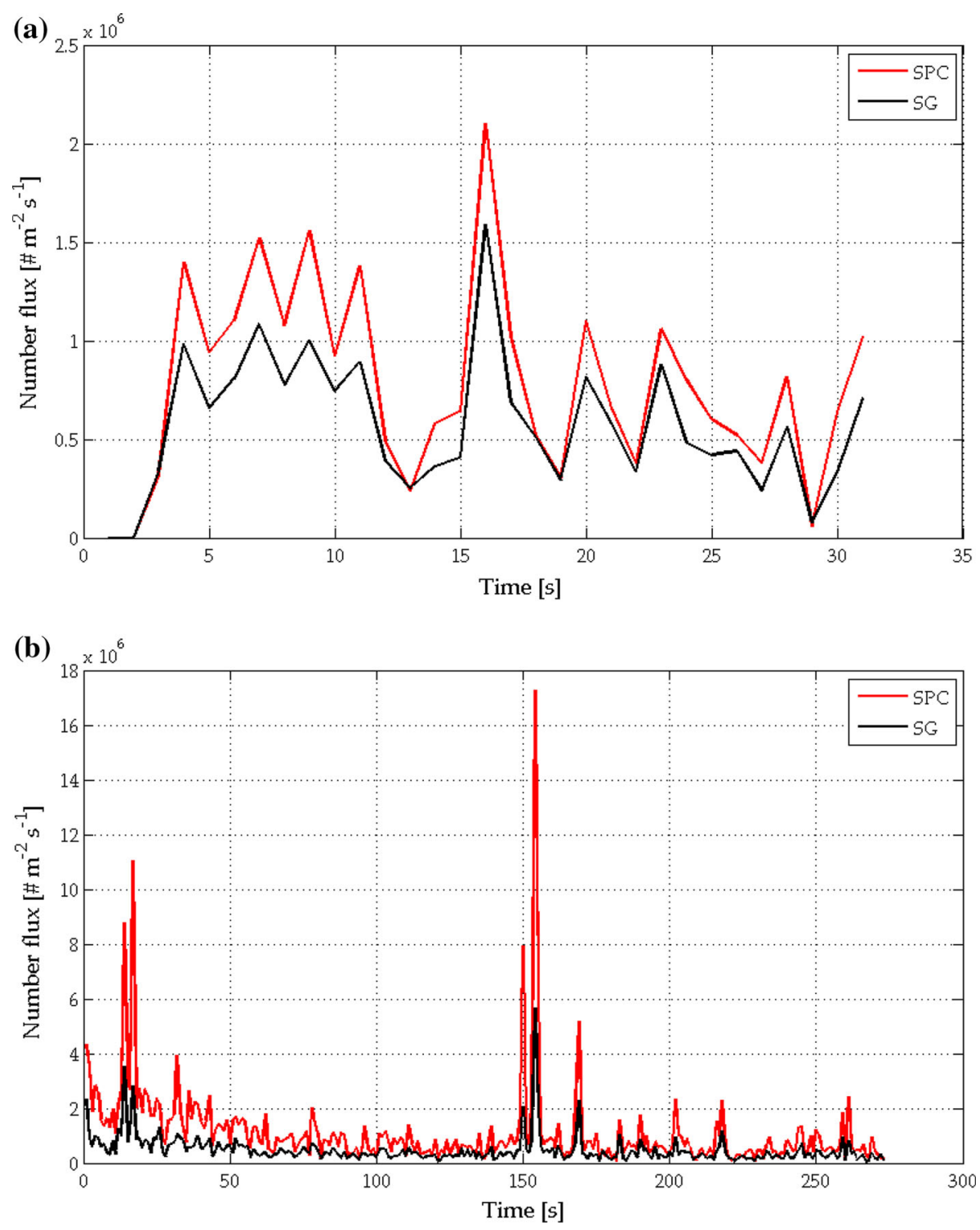

Fig. 15 Number-flux time series signal presented as a 1-s average of the calibration experiment $\mathrm{C} 1$ (a) $(r=$ $0.965)$, and for the drifting experiment $\mathrm{D} 1(\mathbf{b})(r=0.838)$

Similar results as for the calibration experiments were found for the experiments with drifting snow. The correlation for the numbers fluxes was stronger $(r=0.838-0.91)$ compared to the correlation of the mass flux. Other than for the calibration experiment, the ratio of numbers of particles between PTV and SPC was much closer to one (refer to Fig. 15b, $\left.n p_{P T V} / n p_{S P C}=0.854\right)$. 


\section{Discussion}

\subsection{Particle Recognition, Particle Property and Particle Filter}

Our study reveals good agreement in terms of correlation and mass-flux ratio for the two techniques, for both the calibration (Fig. 8) and the drifting snow experiments (Fig. 13). Among the different post-processing parameters that were tested, the particle-recognition threshold shows the largest impact on the results. The smaller this particular threshold is chosen, the more spots in the image are recognized as particles. Consequently, a very small particlerecognition threshold leads to overestimation of the mass flux. As soon as the threshold is large enough, the correlation and the mass-flux ratio are fairly constant, results confirmed by the drifting snow experiments as well. A much clearer picture is observed in the selection of the particle-property filter. As previously described, this filter introduces low-level and highlevel thresholds for the estimation of the particle diameter. The closer together the high-level and the low-level thresholds, the smaller is the ratio between high-level and low-level areas. This affects the estimation of the diameter, and finally the assigned mass of the particle. Being the depth-of-field of a particle proportional to its diameter, this threshold also affects the size of the depth-of-field. More directly, the size of the depth-of-field together with the amount of accepted blurring is further controlled with the third-parameter particle filter.

In the previous section, the optimal threshold for the particle recognition was found to be $50 \%$, for both, the calibration as well as the drifting experiment. This closely matches the settings used by Gromke et al. (2014). For the low-level and high-level particle-property thresholds, we found the optimal thresholds at 40-60\% (calibration) and 45-55\% (drift) respectively (Gromke et al. used 40-60\%). Similarly, the results for the particle-filter threshold were slightly higher in the snow drifting experiment (200\%) compared to the calibration experiment $(175 \%)$. The effect of having a higher particle-property and particle-filter threshold for the drifting experiments when compared to the calibration study is not very significant. For both thresholds the ratio of the mass flux as well as the correlation coefficient reaches a plateau, where the results are minimally affected by the choice of these thresholds (Fig. 13e, f). An explanation for such behaviour is that for larger thresholds, the depth-of-field is greater. Setting the particle-property threshold at $45-55 \%$, the median depth-of-field becomes 27.82 $\mathrm{mm}$, whereas for $40-60 \%$, the median depth-of-field is $18.51 \mathrm{~mm}$ in the drift experiment. The corresponding dimension of the SPC is $25 \mathrm{~mm}$. Having such a large low-level particleproperty threshold, the particle-filter threshold only slightly influences the correlation and the ratio with the mass flux. Only a very small particle filter reduces the depth-of-field, and excludes many particles from fulfilling the criterion.

\subsection{Sensitivity of the Field-of-View}

Analyzing the influence of the dimensions of the field-of-view suggests that a minimum spatial extent of the field-of-view exists, below which the results are affected from excessive undersampling. In the case of the calibration experiment, in which the speed of the particles was relatively slow, a large field-of-view did not reduce the correlation or the ratio between the PTV and SPC signals. This behaviour was observed for both, the streamwise direction as well as the direction perpendicular to the flow (Fig. 10). For the drifting snow experiment, where the average particle speeds were between $5.1 \mathrm{~m} \mathrm{~s}^{-1}$ and $6.9 \mathrm{~m} \mathrm{~s}^{-1}$, the ratio between field-of-view in the streamwise direction and the path length that particles travel between two consecutive images, become much smaller. To obtain the same ratio between particle trajectory and field-of-view, either the field-of-view in the streamwise direction or the sampling 
frequency have to be increased. In our set-up, both of them are limited, the field-of-view due to the finite camera resolution and the sampling frequency due to the limited camera memory. In order to have a long enough experiment, the sampling frequency was chosen as rather low. Although the ratio of field-of-view and particle path is small, the results are similar to those found in the calibration tests. Figure 14b, FOV/path ratio-correlation coefficient, reveals a logarithmic dependence between the dimension of the field-of-view and the correlation between the PTV and SPC mass flux. While the correlation improves with increasing length of the streamwise field-of-view, the results of the mass-flux ratio suggest that a very large field-of-view tends to slightly overestimate the mass flux of the PTV (Fig. 14a) which was not the case in the calibration experiment. This might be an artifact of the too-low sampling frequency. On the other hand, the height of the field-of-view should be at least chosen to be 10 times larger then the height of the SPC, which leads to a vertical field-of-view of about 20 $\mathrm{mm}$. Similarly, an overestimation occurs as an effect of increasing the vertical dimension of the field-of-view, even though the correlation remains constant. The size of the field-of-view, together with the sampling frequency, controls the number of particles that can be detected.

\subsection{Mass Flux, Particle Diameter and Particle Numbers}

The two signals from the two devices in Fig. 7a (calibration experiment C1) are very well correlated. Differences can be due to a bias in the particle size distribution between the two (Fig. 7b). The SPC, although acquiring at high frequency $(40 \mathrm{kHz})$, detects significantly less particles than the PTV. In the calibration experiment, where the snow particles are artificially sieved such that they move through the SPC's and the PTV's field-of-view by gravity only, the particles are homogeneously distributed (Fig. 4). Due to the larger control volume of the PTV (field-of-view $50 \mathrm{~mm} \times 50 \mathrm{~mm} \times$ approx. $30 \mathrm{~mm}$ (maximal depth-of-field)) than the SPC $(25 \mathrm{~mm} \times 2 \mathrm{~mm} \times 0.5 \mathrm{~mm})$, many more particle are detected with the PTV method. A second reason can be found in the particle-size distribution. The graphs show that the SPC detects more smaller particles compared to the PTV method. As the particle-size distribution of the more mono-dispersed sand particles (Fig. 6) shows similar results for SPC and PTV, the lack of resolution at the smallest bin sizes might result in a minor discrepancy between the massflux signals determined with the two instruments. Being only the smallest particle diameters at the origin of this bias the implications for the mass-flux are little. This is supported by the result of the correlation of the particle-number flux (Fig. 15a). By not taking into account the particle diameter, the correlation between SPC and PTV methods was shown to be greater.

Looking at the results of the drifting experiments, we find a more distinguished difference in the particle-size distribution (Fig. 12b) compared to the results of the calibration experiment. We observe that in the drifting experiment, the particle spectrum as detected by the SPC is shifted towards smaller particles. The maximum of the particle diameters is found close to $0.1 \mathrm{~mm}$ for the SPC, while the maximum for the PTV is larger. As a consequence, in all the drifting experiments, the SPC also records many more small particles compared to the PTV method. In case of the drifting experiments additional causes might affect the results of the particle detection from the two instruments.

The profile of the mass flux in the boundary layer is reducing with increasing height above the snow surface, and a peak is observed near the surface. The profile above the peak is reported to be exponential (Sugiura et al. 1998; Nishimura and Nemoto 2005; Guala et al. 2008). Therefore, placing the SPC very close above the surface, where the gradient is high, might introduce a bias in the comparison of the signals of the two instruments. Another difference in the case of the drifting experiment is the previously discussed ratio between the streamwise length of the field-of-view and particle path that is smaller than one. This 
indicates an undersampling of the PTV method which will not capture all particles passing by the field-of-view during the experiment. Considering the high correlation of the particle numbers and the mass flux ratio between SPC and PTV, the chosen sampling frequency is still sufficiently high to capture the time-evolution of drifting particles. A reason causing the differences in the particle-size distribution between calibration and drifting experiments is that it can be assumed that drifting particles show a rapid fragmentation after the initiation of the saltation. The impact of such a behaviour was not investigated within this study.

Therefore, and because the PTV system fails to detect the smallest particles due to its bounded resolution, the differences in the particle-size distribution and the number of detected particles can be explained. Nevertheless, the effect on the mass-flux calculation is smaller since most of the mass is carried by the larger particles. One additional reason that may influences the correlation between the two signals is the SPC's ability of recognizing very small particles. Guala et al. (2008) described that for the very small bins, also the SPC has more difficulties to resolve them compared to particles of the larger bins. Nevertheless this potential effect is hard to estimate.

\subsection{Diameter Interpretation and Spherical Assumption}

A further assumption that needs clarification concerns the interpretation of the diameters by both systems. The SPC detects particles in a diameter range between 0.04 and $0.5 \mathrm{~mm}$, while larger particles are assigned to the $0.5-\mathrm{mm}$ diameter class. For the comparison of the two instruments, the PTV was restricted to the same diameter range and classification. This bounded range may results in an underestimation of the mass flux. As mentioned earlier, both instruments assume the particles to be spherical. Our common experience, further confirmed by Fig. 5, suggests that snow flakes that are not drifted and fragmented, generally are not spherical. Particularly larger snow particles are not well represented by a sphere. Sphericity is also assumed by the SPC. Therefore we assume that the diameter restriction will not lead to a severe underestimation of the mass flux in this study. Furthermore being the two techniques deployed for the study of drifting snow, which implies fragmentation of the snow crystals towards smaller, more spherical geometries, this assumption does not lead to significant errors in the mass flux estimation.

\section{Conclusions}

Wind-tunnel experiments in an ad hoc calibration and typical drifting snow set-up revealed that the mass-flux signals obtained from the two systems are very well correlated. Our study clarified the impact of the post-processing parameters such as particle-recognition, particleproperty and the particle-filter thresholds on the PTV results. The study also demonstrates the need for a proper calibration of the PTV system with an established measurement technique, such as the SPC to be able to correctly measure the drifting snow in a wind tunnel. Figures 8,9 and 13 demonstrate that for all tests in both calibration and drifting experiments, the mass-flux ratio and correlation converge. These results are obtained from tests conducted under different conditions, therefore the validity of the post-processing parameters extends to any drifting snow test in a wind tunnel. The procedure presented can be applied to any other wind-tunnel set-up. The impact of parameters such as sensor size, focal length or aperture was beyond the scope of the study; based on the given equipment and conditions, these parameters were either given or set to fixed values that represented optimal conditions. 
For the particle-diameter distribution, Gromke et al. (2014) mention that diameters detected with a shadowgraphic PTV are generally larger than those reported in the literature from SPC measurements. This reflects the camera's spatial resolution, and confirms our findings that the SPC is more sensitive to smaller particles that may be the result of the fragmentation during drifting. Nevertheless, considering the 1-s averaged signals, the correlation is very strong for optimal settings and thresholds in the acquisition and post-processing (calibration: $r \geq 0.93$, crifting: $r \geq 0.81$ ). Differences in particle numbers and diameter distribution originate from different sampling rates, different control volume sizes, the bounded resolution of the PTV camera compared to the SPC. Uncertainty however remains on differences in the interpretation by the two systems of the diameter of the same snow particle. The results from the test with sand particles, which shows good agreement between SPC and PTV diameter interpretation, suggests that this difference is not large. Due to the fact that very small particles contribute little to the mass flux, the shift in the particle-size distribution only weakly influences the signal of the mass flux. For the present study, the camera's spatial resolution was approximately $47.7 \mu \mathrm{m}$ per pixel, which corresponds approximately to the smallest particle bin size of the SPC. We expect that with a higher resolution, also smaller particles can be detected.

Our results suggest that the PTV technique can be a valid alternative to the more common SPC approach at least in a laboratory environment, and where both the vertical and streamwise components of the mass flux are needed, as well as higher sampling frequency. The applicability of the set-up to field studies poses several technical issues and needs to be demonstrated. SPC limits are known, while PTV's limits depend on the camera and a general conclusion on this is too restrictive.

Acknowledgements The authors wish to express their acknowledgment to the Swiss National Science Foundation (SNF) for funding this research (R grant number 200021_147184) as well as to Christof Gromke and the SNF (REquip grant number 206021_133786) for installing the PTV equipment. We further thank Prof. Kouichi Nishimura for his support in operating the SPC, Matthias Jaggi for his contribution concerning electronics issues, the SLF workshop team that helped to prepare all the hardware at the wind tunnel as well as the reviewers for their valuable and constructive input.

\section{References}

Bagnold R (1935) The movement of desert sand. Geogr J 85(4):342-365

Bagnold RA (1941) The physics of wind blown sand and desert dunes. Methuen, London, $256 \mathrm{pp}$

Burri K, Gromke C, Lehning M, Graf F (2011) Aeolian sediment transport over vegetation canopies: a wind tunnel study with live plants. Aeolian R 3(2):205-213. doi:10.1016/j.aeolia.2011.01.003

Clifton A, Rüedi JD, Lehning M (2006) Snow saltation threshold measurements in a drifting-snow wind tunnel. J Glaciol 52(179):585-596. doi:10.3189/172756506781828430

Clifton A, Manes C, Rüedi JD, Guala M, Lehning M (2008) On shear-driven ventilation of snow. BoundaryLayer Meteorol 126(2):249-261. doi:10.1007/s10546-007-9235-0

Doorschot JJ, Lehning M, Vrouwe A (2004) Field measurements of snow-drift threshold and mass fluxes, and related model simulations. Boundary-Layer Meteorol 113(3):347-368. doi:10.1007/s 10546-004-8659-z

Gordon M, Taylor PA (2009) Measurements of blowing snow, part i: particle shape, size distribution, velocity, and number flux at Churchill, Manitoba, Canada. Cold Reg Sci Technol 55(1):63-74. doi:10.1016/j. coldregions.2008.05.001, http://www.sciencedirect.com/science/article/pii/S0165232X08000736

Gordon M, Savelyev S, Taylor PA (2009) Measurements of blowing snow, part ii: mass and number density profiles and saltation height at Franklin bay, NWT, Canada. Cold Reg Sci Technol 55(1):75-85. doi:10.1016/ j.coldregions.2008.07.001, http://www.sciencedirect.com/science/article/pii/S0165232X0800102X

Gromke C, Manes C, Walter B, Lehning M, Guala M (2011) Aerodynamic roughness length of fresh snow. Boundary-Layer Meteorol 141(1):21-34. doi:10.1007/s10546-011-9623-3 
Gromke C, Horender S, Walter B, Lehning M (2014) Snow particle characteristics in the saltation layer. J Glaciol 60(221):431-439. doi:10.3189/2014JoG13J079

Groot Zwaaftink C, Cagnati A, Crepaz A, Fierz C, Macelloni G, Valt M, Lehning M (2013) Event-driven deposition of snow on the antarctic plateau: analyzing field measurements with snowpack. The Cryosphere 7(1):333-347. doi:10.5194/tc-7-333-2013

Guala M, Manes C, Clifton A, Lehning M (2008) On the saltation of fresh snow in a wind tunnel: profile characterization and single particle statistics. J Geophys Res: Earth Surface (2003-2012) 113(F3), doi:10. 1029/2007JF000975

Kim KS, Kim SS (1994) Drop sizing and depth-of-field correction in tv imaging. At Sprays 4(1):65-78. doi:10. 1615/AtomizSpr.v4.i1.30

Kimura MTIT T (1993) Design and making of spc-vii p pp. 665-670 (in Japanese)

LaVision-GmbH (2011) Product-manual for davis 8.0 pp 9-46

Nishimura K, Nemoto M (2005) Blowing snow at mizuho station, antarctica. Philos Trans Roy Soc 363(1832):1647-1662. doi:10.1098/rsta.2005.1599

Nishimura K, Sugiura K, Nemoto M, Maeno N (1998) Measurements and numerical simulations of snowparticle saltation. Ann Glaciol 26:184-190

Paterna E, Crivelli P, Lehning M (2016) Decoupling of mass flux and turbulent wind fluctuations in drifting snow. Geophys Res Lett pp n/a-n/a. doi:10.1002/2016GL068171, http://dx.doi.org/10.1002/ 2016GL068171, 2016GL068171

Pokorny M, Horender S (2014) Measurement of particle rotation in a saltation layer. Earth Surf Process Landf 39(13):1803-1811. doi:10.1002/esp.3568

Schirmer M, Lehning M, Schweizer J (2009) Statistical forecasting of regional avalanche danger using simulated snow-cover data. J Glaciol 55(193):761-768. doi:10.3189/002214309790152429

Schmidt R (1977) A system that measures blowing snow. Rocky Mountain Forest and Range Experiment Station, Forest Service, US Department of Agriculture

Schmidt R (1986) Transport rate of drifting snow and the mean wind speed profile. Boundary-Layer Meteorol 34(3):213-241. doi:10.1007/BF00122380

Sugiura K, Nishimura K, Maeno N, Kimura T (1998) Measurements of snow mass flux and transport rate at different particle diameters in drifting snow. Cold Reg Sci Technol 27(2):83-89. doi:10.1016/ S0165-232X(98)00002-0

Walter B, Gromke C, Lehning M (2012a) Shear-stress partitioning in live plant canopies and modifications to raupachs model. Boundary-Layer Meteorol 144(2):217-241. doi:10.1007/s10546-012-9719-4

Walter B, Gromke C, Leonard K, Clifton A, Lehning M (2012) Spatially resolved skin friction velocity measurements using irwin sensors: a calibration and accuracy analysis. J Wind Eng Ind Aerodyn 104:314321. doi:10.1016/j.jweia.2012.02.018

Walter B, Gromke C, Leonard KC, Manes C, Lehning M (2012c) Spatio-temporal surface shear-stress variability in live plant canopies and cube arrays. Boundary-Layer Meteorol 143(2):337-356. doi:10.1007/ s10546-011-9690-5

Walter B, Horender S, Voegeli C, Lehning M (2014) Experimental assessment of owen's second hypothesis on surface shear stress induced by a fluid during sediment saltation. Geophys Res Lett 41(17):6298-6305. doi:10.1002/2014GL061069 